\title{
Frontal Lobe Ependymal Tumor
}

National Cancer Institute

\section{Source}

National Cancer Institute. Frontal Lobe Ependymal Tumor. NCI Thesaurus. Code C131573.

An ependymal tumor affecting the frontal lobe of the brain. 